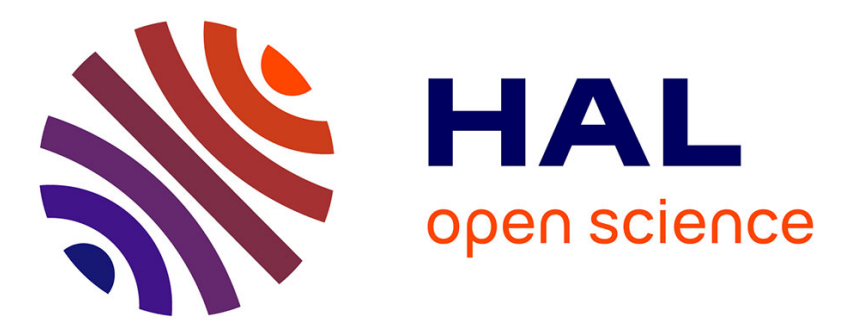

\title{
Impact of fast ions on a trapped-electron-mode dominated plasma in a JT-60U hybrid scenario
}

S. Mazzi, David Zarzoso, J. Garcia, T. Görler, A. Di Siena, Yann Camenen, S. Benkadda, M. Yoshida, N. Hayashi, K. Shinohara

\section{- To cite this version:}

S. Mazzi, David Zarzoso, J. Garcia, T. Görler, A. Di Siena, et al.. Impact of fast ions on a trapped-electron-mode dominated plasma in a JT-60U hybrid scenario. Nuclear Fusion, 2020, 60 (4), pp.046026. 10.1088/1741-4326/ab74a1 . hal-02989858

\section{HAL Id: hal-02989858 https://hal.science/hal-02989858}

Submitted on 5 Nov 2020

HAL is a multi-disciplinary open access archive for the deposit and dissemination of scientific research documents, whether they are published or not. The documents may come from teaching and research institutions in France or abroad, or from public or private research centers.
L'archive ouverte pluridisciplinaire HAL, est destinée au dépôt et à la diffusion de documents scientifiques de niveau recherche, publiés ou non, émanant des établissements d'enseignement et de recherche français ou étrangers, des laboratoires publics ou privés. 


\title{
Impact of Fast Ions on a Trapped-Electron-Mode Dominated Plasma in a JT-60U Hybrid Scenario
}

\author{
S. Mazzi ${ }^{1,2}$, D. Zarzoso ${ }^{1}$, J. Garcia ${ }^{2}$, T. Görler ${ }^{3}$, A. Di Siena ${ }^{3}$, Y. \\ Camenen $^{1}$, S. Benkadda ${ }^{1}$, M. Yoshida ${ }^{4}$, N. Hayashi ${ }^{4}$, K. \\ Shinohara ${ }^{4}$. \\ 1 Aix-Marseille Université, AMU, PIIM, UMR 7345 Marseille, France \\ 2 CEA, IRFM, F-13108 Saint-Paul-lez-Durance, France \\ 3 Max Planck Institute for Plasma Physics, Boltzmannstr. 2, 85748 Garching, Germany \\ ${ }^{4}$ National Institutes for Quantum and Radiological Science and Technology, Naka, \\ Ibaraki 311-0193, Japan
}

\begin{abstract}
The impact of fast ions on a trapped electron mode (TEM) is extensively analysed by linear and nonlinear gyrokinetic simulations for a JT-60U plasma at high $\beta$ and low magnetic shear using the GENE code in local approximation. For the first time, it is shown that TEM-induced turbulent transport may remain unaffected by the steep fast ion pressure profile generated by the Neutral Beam Injection. Unlike recent observations of ion temperature gradient (ITG)-induced turbulent transport reductions due to fast ions, TEMdominated systems could act differently in the presence of a significant fast ion population. The possible role of zonal flows as a saturation mechanism is analyzed, showing that their weak impact in the reported JT-60U scenario might lead to different behaviour of fast ions with respect to the ITG-dominated discharges. It is also shown that Alfvénic shear modes are destabilized at low wave numbers $(n \simeq 8)$. They are identified as drift Alfvén waves destabilized by ITG, which is a form of Alfvénic ITG instabilities. Deep numerical analysis provides the physical parameter range in which ITG-driven BAEs are stabilized. These results open the way to new possibilities of tailoring future experimental scenarios in order to benefit from transport reduction by fast ions.
\end{abstract}




\section{Introduction}

In present fusion devices, heating systems, such as neutral beam injection (NBI) and ion cyclotron resonance heating $(\mathrm{ICRH})$, provide long tails in the distribution function which represent the fast ion population. Fast ions have energies larger than those of the thermal particles. In future devices, primarily in ITER D-T scenarios, $\alpha$-particles are expected to be the main heating mechanism. Both, fusion generated and externally heated fast ions must be well confined in order to transfer their energy to the thermal part of the plasma through Coulomb collisions, and self-sustain the fusion reaction. Furthermore, fast particle losses can damage the plasma facing components and thus limit the operating life of the internal wall. Energetic particles can also excite the so-called energetic particle driven modes (EPM) which can increase the energetic particle transport from the core to the edge of the tokamak resulting eventually in significant losses, even in the presence of one single electrostatic mode [1]. In addition, steep pressure profiles lead to microinstabilities, which in nonlinear phase result in microturbulence. Turbulent transport and energetic particles losses can dramatically reduce the overall performance of the device (see e.g. [2,3] and references therein). Therefore, analyzing the mutual interaction between energetic particles and microturbulence is of prime importance towards ITER steady state operational scenarios.

Recently these analyses have been started in both linear and nonlinear regimes by means of computationally expensive gyrokinetic simulations motivated by experimental evidences of fast ion turbulence reduction. In linear regimes, some efforts have been dedicated to explain the fast ion turbulence reduction through the dilution mechanism of thermal ion drive $[4,5]$ and through the shift in phase-space of the wave-particle resonance when introducing fast ions [6]. In nonlinear regimes, a comprehensive framework has not been established yet, though some insights have been gained [7]. Nonlinear electromagnetic gyrokinetic simulations have been able to reproduce the fast ion turbulence stabilization in ITG-dominated JET [7-12] and ASDEX-Upgrade (AUG) [13] discharges. Such stabilization might be also significant in the presence of $\alpha$-particles in ITER D-T plasmas [14]. Unfortunately, reduction of turbulence by fast ions might be limited by the destabilization of EPMs driven by the gradients of the distribution function in phase space. Indeed, although fast ions have been observed to linearly reduce the turbulence level in electrostatic regimes [15], they can subsequently excite EPMs and fast-ion driven instabilities in the Alfén frequency range, which can transfer nonlinearly energy to turbulent modes $[15,16]$. In this case, fast and thermal ion transport would be sharply increased, limiting the contribution of the suprathermal particles to the ITG stabilization.

One of the key questions is whether the turbulence reduction obtained in JET and AUG is universal or it is just restricted to those tokamaks and ITG-dominated electromagnetic turbulence regimes. Elucidating this question is essential in order to give confidence on the predictions performed for future tokamaks with a significant fast ion fraction, such as ITER or JT-60SA [17]. Therefore, in this paper we analyse a scenario similar to the one analysed in the past: the so-called JET hybrid H-mode scenario, at high $\beta$ and low core magnetic shear. The difference comes from the drive of the background turbulence. For that purpose, a JT-60U discharge with the characteristics of the JET hybrid scenario has been chosen [18], with sufficiently strong density gradient to induce Trapped Electron Mode (TEM) turbulence [19].

In this paper, we perform extensive numerical analysis with the Gene gyrokinetic code [20]. The following studies have been performed in a quite unique framework that includes fast ion particles, turbulent microinstability induced transport and high $\beta$, leading to a very complex and computationally demanding system. The paper is organized as follows: in section 2, the simulation setup is reported and the choices for the numerical input parameters are briefly discussed. Section 3 presents the relevant results of the performed linear simulations, introducing the preliminary physical results provided by the linear spectra of the simulations. A major effort is employed in analysing the linear impact of the fast ions on the system. Section 4 addresses the nonlinear impact of fast ions on TEM, but it also reviews the nonlinear behaviour of the linearly excited low- $k_{y}$ modes. Conclusions are drawn in section 5 . 


\section{GENE Simulation Setup}

All the following numerical simulations are performed by means of the $\delta f$ gyrokinetic code GENE [20], which solves the gyrokinetic Vlasov equation coupled to Poisson's (or quasineutrality condition) and Ampere's equations with also parallel magnetic fluctuations. GENE exploits a non-Cartesian coordinate system, namely the field-aligned system, where the three spatial coordinates are defined as: $x$ is the radial coordinate, orthogonal to the magnetic field line and to the magnetic surface, $y$ is the binormal direction, normal to the field line but tangent to the magnetic surface, and finally $z$ is the coordinate running along the magnetic field line. All the simulations have been performed in the local approach, i.e. only a flux-tube has been taken into account, which is justified by the small Larmor radius compared to the minor radius of the JT-60U device $-\rho_{i}^{*} \approx 1 / 200$ and $\rho_{i}^{*} \approx 1 / 400$ for the thermal deuterium and carbon impurities respectively, whereas $\rho_{\text {fast }}^{*} \approx 1 / 100$ for the fast ions. Further check on the validity of the local approach for this JT-60U hybrid discharge have been performed and reported in Appendix A. Collisions have been taken into account in all the performed simulations, and a linearized LandauBoltzmann collisional operator has been employed. The plasma equilibrium used in the simulations has been calculated by means of the HELENA code [21], on the basis of integrated modelling simulations performed with CRONOS suite of codes [22]. For the Gene analysis, the time $t=27 \mathrm{~s}$ has been selected as it corresponds to the stationary phase of the JT-60U hybrid scenario with NBI heating discharge \#48158 [18]. The flux-tube simulated is localized at a normalized toroidal flux coordinate $\rho=0.33$, since it is a representative position of the core microturbulence for hybrid regimes. Two different equilibria have been employed in GENE simulations, depending on whether the fast particles were considered or not. Indeed, if the fast ion pressure was neglected, the equilibrium was recalculated with only the thermal part of the total pressure. The slowing down distribution has been calculated by means of the OMFC Monte-Carlo code [23]. The fast ions are retained as Fast Deuterium (FD), whereas the thermal plasma is composed of Electron, Deuterium and impurity (Carbon) species. The fast ion distribution considered in this paper is a maxwellian equivalent to the slowing down distribution function.

Concerning the nonlinear simulations, perpendicular box size has been chosen as $\left[L_{x}, L_{y}\right]=[812,125]$ in units of thermal ion gyroradius with respect to sound speed, perpendicular grid discretisation as $\left[n_{x}, n_{k y}\right]=$ $[864,32], 32$ point discretisation in parallel direction, 48 points in parallel velocity direction and 12 in magnetic moment direction. In the case fast ions are not taken into account, the box size has been decreased to $\left[L_{x}, L_{y}\right]=[622,125]$, because of the smaller gyroradius of the thermal part of the plasma, but the perpendicular grid discretisation has been increased to $\left[n_{x}, n_{k y}\right]=[1152,32]$. Such high values of $L_{x}$ and $n_{x}$ were required in order to fulfil the boundary conditions in the low magnetic shear equilibria, as pointed out in table 1. Extensive convergence tests have been carried out for validating the resolution chosen. At this regard, the reader must note again the low value of the magnetic shear $(\hat{s}=0.05)$, which increases the distance between consecutive rational surfaces $\delta q$, since $\delta q=x /(q \hat{s} n)$, where $x$ is the radial coordinate and $n$ the toroidal wavenumber. It could affect the ballooning representation, assumed in the Gene code. However, dedicated studies about the validity of ballooning representation for low magnetic shear systems will be carried out in a separate work.

The linear turbulent growth rate is in units of $c_{s} / a$ where $c_{s}=\sqrt{T_{e} / m_{i}}$ with $T_{e}$ the electron temperature and $m_{i}$ the thermal ion mass, and $a$ is the minor radius.

It should be reported that, in this discharge a low torque by NBI was applied. Therefore, the toroidal rotation and especially the $E \times B$ shearing are quite low and they have not been taken into account in the simulations performed in this paper.

\section{Linear Simulations}

In this section the results of the linear simulations of JT-60U discharge \#48158 are presented.

Table 1: Input parameters derived from the JT-60U Hybrid Scenario 48158 discharge [18] at $\rho=0.33$. All the parameters missing in this table are reported in table 2 , where the modifications among the different cases studied have been detailed. $\epsilon=a / R$ is the inverse aspect ratio, $\hat{s}$ is the magnetic shear, $q$ the safety factor and $\nu^{*}$ is the normalized collision frequency defined as $\nu^{*} \equiv \frac{a n_{e}}{4|e|^{2} n_{i}} \nu_{e i}$ where $\nu_{e i}$ is the electron-ion collision rate of Hinton and Hazeltine [24]. For completeness, $T_{e}=3.44 \mathrm{keV}$ and $B=1.46 \mathrm{~T}$.

\begin{tabular}{cc}
\hline Parameter & Value \\
\hline$R / L_{T_{e}}$ & 7.4 \\
$R / L_{n_{e}}$ & 5.28 \\
$R / L_{T_{\text {carb }}}$ & 7.73 \\
$T_{i} / T_{e}$ & 1.22 \\
$T_{\text {fast }} / T_{e}$ & 8.62 \\
$\epsilon$ & 0.3 \\
$\hat{s}$ & 0.05 \\
$q$ & 1.06 \\
$\nu^{*}$ & $8.5 \cdot 10^{-5}$ \\
\hline
\end{tabular}




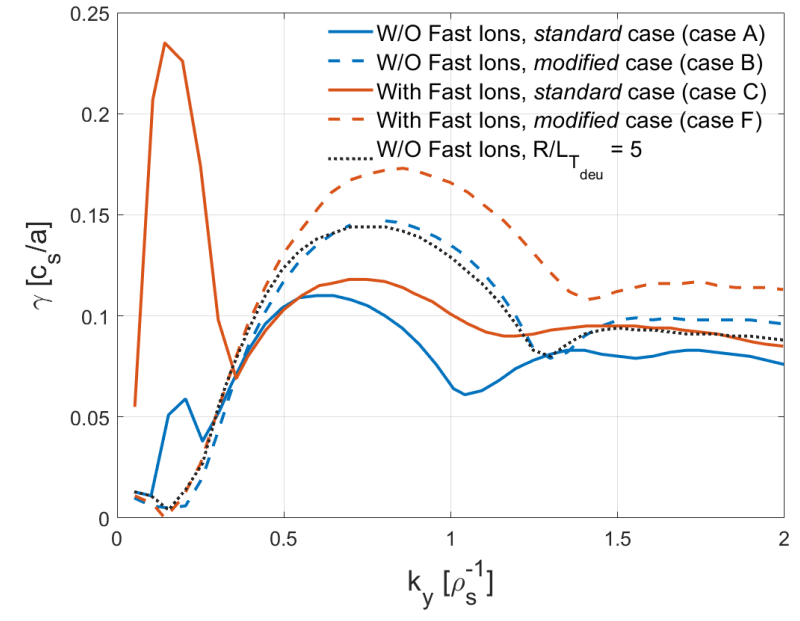

(a)

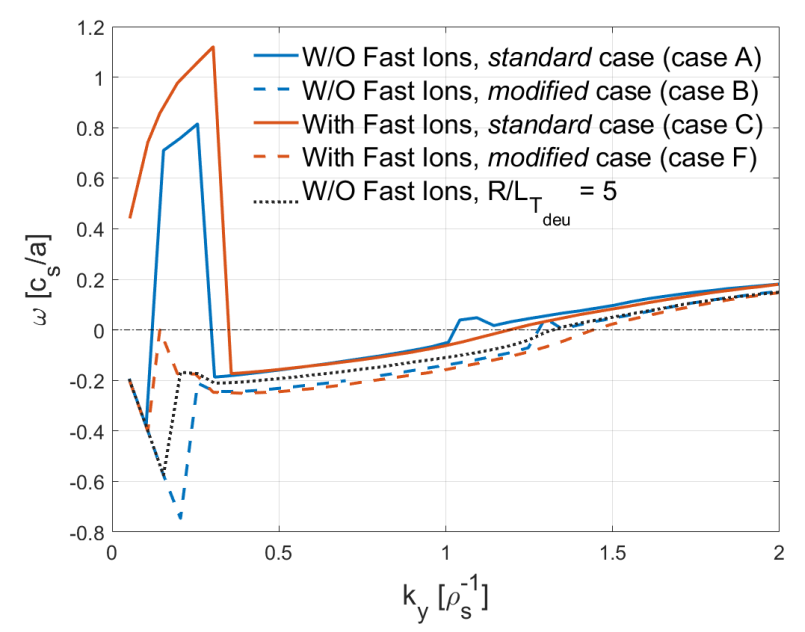

(b)

Figure 1: Linear growth rates (a) and frequencies (b) for the JT-60U 48158 discharge.

In figures 1(a) and (b), the linear growth rates and frequencies are shown respectively for different kinds of simulation of JT-60U 48158 discharge. They are plotted against the binormal wavenumber $k_{y}$, normalized to the thermal ion gyroradius $\rho_{s}$ with respect to the sound speed. In table 2 , the whole set of input parameters for the different simulation cases is listed.

\subsection{Linear Spectra Analysis}

In this subsection, we will introduce and analyse the linear spectra, obtained through a scan over the binormal wave vectors in the Fourier space. It is possible to subdivide the spectrum in three different regions: the first one is limited to $k_{y} \leq 0.35$, often named in the following as low- $k_{y}$ region; in the second region (roughly going from $k_{y}=0.35$ to $k_{y}=1.2$ ) the TEM instability dominates; finally, the small scale region shows a non-global dominant instability which stabilizes around $k_{y}=5$. Moreover, it is important to say that no sign of ETG excitation has been found up to $k_{y} \rho_{s}=70$.

The solid blue profile of figure 1(a) represents the growth rate of the most unstable modes without including fast ions (case A), in which the dominant instability has a broad maximum peaking around $k_{y}=$ 0.6. Characterizing and identifying the most dominant mode is crucial in order to figure out the impact of the fast ions on the system studied. In figures 2(a) and (b) relevant analyses in order to identify the bulkinstability have been performed: the contribution of each species to the time trace of the time derivative of the potential energy and the energy exchange in the velocity space are shown, respectively, at $k_{y}=0.6$ for the nominal input parameter simulation in which the fast ions are not included (the solid blue profile in figure 1, i.e. case A). Indeed, in GENE it is possible to investigate the direction and the magnitude of the energy exchange for each species taken into account. In this context, this could be done through the study of the system free energy $E_{f e}$, which is defined as the sum of the kinetic $E_{k}$ and field $E_{w}$ contributions [25]. Note that the field energy $E_{w}$ is also called potential energy in the following. From the free energy balance relation in the steady-state regime, it is possible to evaluate the rate of energy transferred from the particles to the wave $\left(\partial E_{w} / \partial t\right)$ and, in the opposite direction, the wave contribution to the energy of the particles $\left(\partial E_{k} / \partial t\right)$. Therefore, this implies that a linear growth rate could be defined as

$\gamma=\sum_{s} \gamma_{s}=\frac{1}{E_{w}} \sum_{s} \frac{\partial E_{w, s}}{\partial t}$

where the subscript $s$ refers to each species. From definition 1, it is possible to determine the contribution of each species to the resulting linear growth rate. This is shown in figure 2(a). Furthermore, we are allowed to extract the dependence of $\gamma_{s}$ on the velocity component of the phase space and plot it in the velocity space, which is done in figure $2(\mathrm{~b})$.

Thereby, the electron species mainly excites the instability at the wavenumber analyzed now, as well as the thermal deuterium does in a lighter way. This latter consideration could be an indicator of a not-pure TEM. Conversely, Carbon impurities are damping the mode. Indeed, from figure 2(b), it appears clearly that the particles responsible for the excitation of the instability are localized within the trapping cone marked in the figure by a dashed line. Hence, we 
Table 2: Parameters for the JT-60U Hybrid Scenario 48158 discharge [18] at $\rho=0.33$. In the table, the relevant differences among the various cases studied are represented. Here, $n$ represents the density normalized to the electron species one, $R / L_{T, n}$ the normalized logarithmic temperature and density gradient for different species, $\beta_{e}$ the electron-beta. All the other significant parameters of the discharge are summarized in table 1.

\begin{tabular}{ccccccccc}
\hline & case & $R / L_{T_{i}}$ & $R / L_{n_{i}}$ & $n_{\text {deu }}$ & $n_{\text {fast }}$ & $R / L_{T_{\text {fast }}}$ & $R / L_{n_{\text {fast }}}$ & $\beta_{e}[\%]$ \\
\hline $\mathrm{A}$ & standard - W/O Fast Ions & 7.73 & 5.28 & 0.44 & - & - & - & 1.48 \\
$\mathrm{~B}$ & modified $-\mathrm{W} / \mathrm{O}$ Fast Ions & 6.21 & 5.28 & 0.34 & - & - & - & 1.2 \\
$\mathrm{C}$ & standard $-\mathrm{W}$ Fast Ions & 7.73 & 7.16 & 0.3 & 0.135 & 2.08 & 4.53 & 1.48 \\
$\mathrm{D}$ & standard $-65 \% \nabla P_{\text {fast }}$ - W Fast Ions & 7.73 & 7.16 & 0.3 & 0.135 & 0.17 & 2.77 & 1.48 \\
$\mathrm{E}$ & standard $-80 \% \nabla P_{\text {fast }}$ - W Fast Ions & 7.73 & 7.16 & 0.3 & 0.135 & 0.17 & 1.14 & 1.48 \\
$\mathrm{~F}$ & modified $-50 \% \nabla P_{\text {fast }}$ - W Fast Ions & 6.21 & 7.16 & 0.23 & 0.11 & 0.84 & 2.77 & 1.2 \\
$\mathrm{G}$ & modified $-80 \% \nabla P_{\text {fast }}$ - W Fast Ions & 6.21 & 7.16 & 0.23 & 0.11 & 0.17 & 1.14 & 1.2 \\
\hline
\end{tabular}

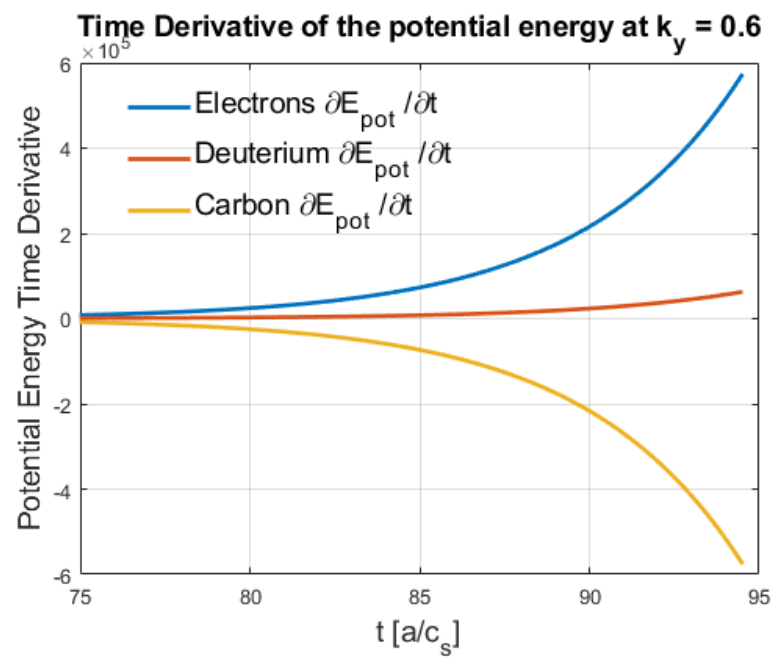

(a)

Figure 2: (a) Contribution of each species to the potential energy time derivative and (b) the electron curvature term contribution to the overall linear growth rate in velocity space at $k_{y}=0.6$, i.e. at the position at which the peak of the TEM has been detected, for the electromagnetic system without fast ions (case A).

can state that TEM is the bulk-instability, which is consistent with respect to the negative sign of the frequency in the spectrum.

Concerning the long tail of the growth rate spectrum of this JT-60U discharge, the modes are driven mainly by the carbon impurities. It is well known that the ITG instability is excited at lower $k_{y}$ - in the normalization here used, around $k_{y} \simeq 0.5$ for what concerns deuterium-ITG. The large value of the impurity temperature gradient likely destabilizes carbon-ITG instabilities in these numerical analyses. Indeed, this carbon-ITG mode peaks around $k_{y}=1.5$. The shift to the right in the spectrum is consistent with the decreasing of the carbon gyroradius with respect to the deuterium one, due to the greater carbon mass

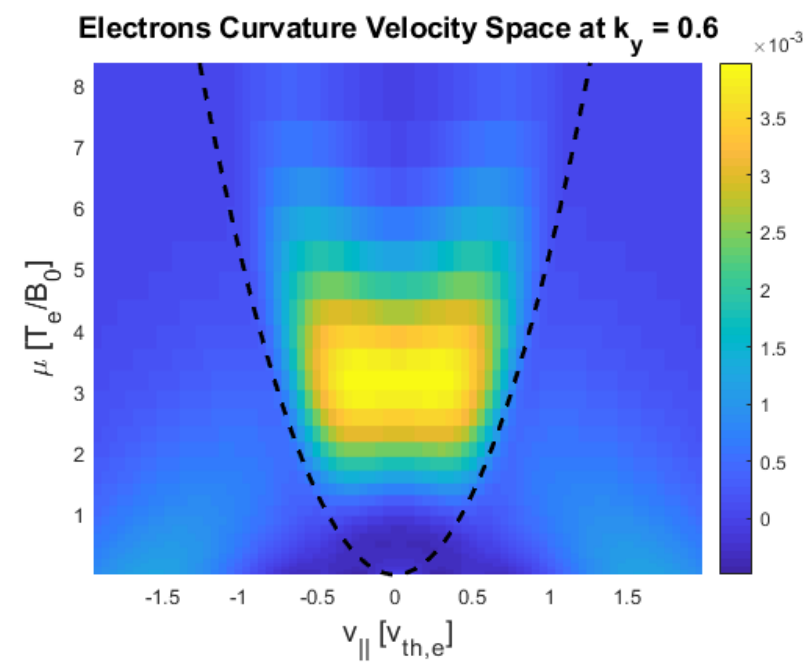

(b)

than deuterium one, which leads to a slower thermal velocity for carbon. Moreover, the species contribution to the overall growth rate analysis, the same employed in figure $2(\mathrm{a})$, is performed also for $k_{y}=1.5$, revealing that carbon impurities are destabilizing the mode. In figure 3 , it is also relevant to notice the electromagnetic stabilization of the carbon-ITG mode occurring in this high- $k_{y}$ region, which is a well-known effect [26,27]. The significant amount of impurities influences the linear growth rates, because of the carbon charge which increases the collisionality among species, also in the TEM region. Indeed, carbon impurities lead to a stabilization of the TEM instability of figure 1(a), likely due to detrapping of electrons (for sake of simplicity, this effect is not directly shown in a plot). 


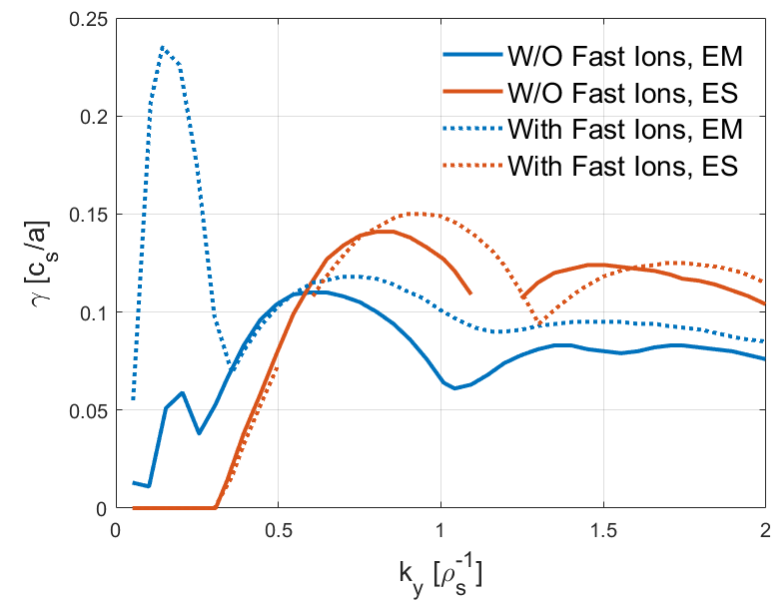

Figure 3: Linear growth rates for the JT-60U 48158 discharge. A comparison among the electromagnetic and electrostatic standard cases, with (case C) and without (case A) fast ions, is shown.

The linear electrostatic simulation without fast ions for the standard case (case A) has been performed and is represented by the solid red profile in figure 3 . Comparing the EM and ES cases, the growth rate is enhanced when magnetic fluctuations are neglected, except for the low- $k_{y}\left(k_{y}<0.3\right)$ region, and a slight shift of the instabilities to small scales occurs.

Looking at the low- $k_{y}$ region of figure 1, it clearly appears that an instability is excited for what concerns the standard system in which fast ions are not included (case A). This mode is localized around a small range of wavenumbers, and its growth rate is low with respect to the bulk-instability. The electrostatic simulation appears to be completely stable in the same region, as figure 3 shows. Therefore, we can conclude that the instability is electromagnetic.

The same analysis of figure 2(a) has been carried out also for this instability, revealing that the thermal ions are driving the mode, nonetheless for sake of conciseness the plots have not been reported. Also, in figures 4(a) and (b), a scan over the thermal ion species temperature gradient is computed. The vertical red dashed line represents the nominal value as it has been calculated by CRONOS modelling, namely $R / L_{T}=7.725$. A transition from an electrondriven mode to the already mentioned deuteriumdriven instability beyond $R / L_{T}=6.5$, characterized by a sharp increase of the linear growth rates for the increase of the temperature gradient and also a shift from negative to positive frequencies of the dominant mode are observed.

Eventually, the deuterium-driven instability arising around $k_{y}=0.2$ has been identified as a shearAlfvénic Ion Temperature Gradient mode (AITG)

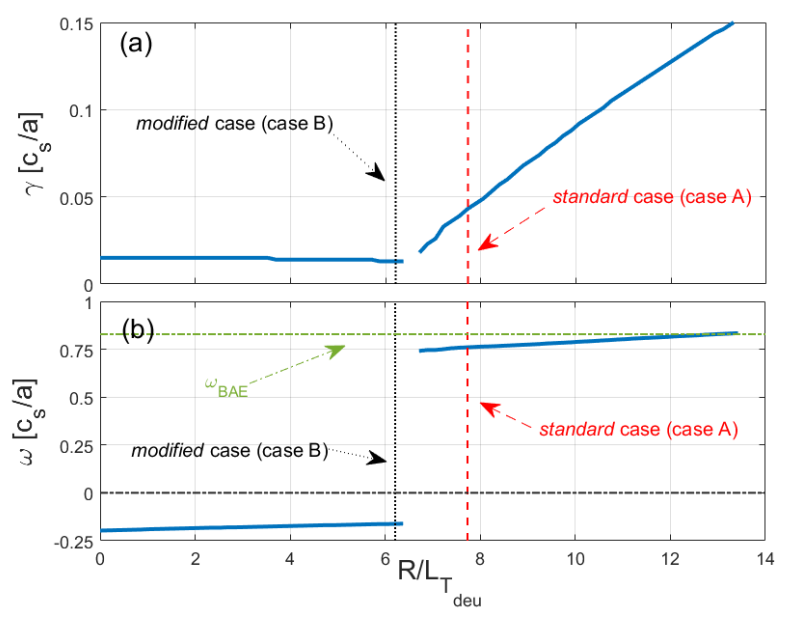

Figure 4: (a) Growth rate and (b) frequency at $k_{y}=0.2$, as a function of deuterium temperature gradient in the system without fast ions (EM simulations). Note that the vertical dashed red lines represents the nominal thermal deuterium temperature gradient (case A), whereas the black dotted lines the modified one (case B). The horizontal green line represents the frequency for the GAM/BAE range calculated through relation 2 .

$[28,29]$, which will be discussed further in this section. This mode has been already detected in global gyrokinetic simulations [30], and also clear experimental evidences have been recently presented [31]. AITG modes are localized electromagnetic instabilities driven by the thermal ion temperature gradient when the system is sufficiently close to the ideal MHD marginal stability. It could be considered as an unstable branch connecting the Kinetic Ballooning Mode (KBM) [32] and the Beta-induced Alfvén Eigenmode (BAE) [33], since AITG could be excited below the ideal MHD $\beta$ limit. AITG and KBM branches could be distinguished from the destabilization mechanism. Indeed, AITG is destabilized through a kinetic resonance (driven then by the ion temperature gradient), whereas KBM is not resonant but it has a fluid-like destabilization. Moreover, the resonant effect is maximum when BAE and KBM branches are strongly coupled [34]. This occurs when the relation $\Omega_{* p i}^{2} \equiv\left(\omega_{* p i} / \omega_{t i}\right) \simeq(7 / 4+\tau) q^{2}$ is verified, where $\omega_{* p i}=\left(T_{i} / e B\right) k_{y}\left(\nabla \ln n_{i}\right)\left(1+\eta_{i}\right)$, $\eta_{i}=\nabla \ln T_{i} / \nabla \ln n_{i}, \omega_{t i}=\sqrt{\left(2 T_{i} / m_{i}\right) /\left(R_{0} * q\right) \text { and }}$ $\tau=T_{e} / T_{i}$ [34]. According to our parameters, this latter relation holds almost perfectly. The stability of the AITG mode is influenced by many physical parameters such as the magnetic shear and the pressure gradients. It has been seen that it is destabilized in weak magnetic shear and low pressure gradient plasmas at low- $n$ toroidal mode number [31]. As table 2 shows, the considered magnetic shear of this JT-60U Hybrid 
Scenario is $\hat{s}=0.05$, whereas the pressure gradient in the case without fast ions (case A) is $\alpha \approx 0.9$, where $\alpha=-R_{0} q^{2} d \beta / d r$ with $d \beta / d r$ derived from $R / L_{T}$ and $R / L_{n}$. In [31], AITGs are found to be destabilized already at smaller pressure gradient values, but the growth rate trend implies that they are more unstable at higher $\alpha$ [29]. Furthermore, the electrostatic potential $\phi$ structure is wide along the parallel direction, which is in good agreement with the results found in GENE simulations of this JT-60U discharge. Moreover, when $\alpha$ is increased, a shift to higher toroidal wavenumbers is also reported. This latter consideration enforces our instability identification, since GENE detected the excitation of this AITG mode at $n=6$, where $n$ is the toroidal wavenumber, which is reflected in the experimental measurements and numerical simulations documented in Ref. [31]. In the latter reference, AITG has been measured by multiple diagnostics lying in the frequency range $15-40 \mathrm{kHz}$, which is in good agreement with the frequency calculated by our numerical simulations. It must be noted however, that in Ref. [31], the rational surface $q=1$ was present, whereas in the simulations presented here, $q=1.06$.

When fast ions are retained in the simulations (case C), the growth rate spectrum is strongly modified in the narrow $k_{y}<0.3$ region. A very high spike appears for the electromagnetic simulation and peaks around $k_{y}=0.15$ and the corresponding mode frequency is in the GAM range, as determined by the equation (16) in Ref. [35]:

$\omega_{G A M}^{2}=\left(\frac{2}{\tau}+\frac{7}{2}+\frac{1}{\tau q^{2}}\right)\left(\frac{c_{s i}}{R}\right)^{2}$

where $\tau=T_{i} / T_{e}$ and $c_{s i}=\sqrt{T_{i} / m_{i}}$. This is also reported in figure 4(b), where one can appreciate a good agreement between $\omega_{G A M}$ and the mode frequency. Nevertheless, $\omega_{G A M}=\omega_{B A E}$ only assuming that $k_{\|}=n q-m=0$ holds. This is demonstrated in figure $\mathrm{A} 1$, where one can see that the mode peaks at the central rational surface, therefore $k_{\|}=0$.

Actually, when the EM fluctuations are not taken into account, the instability totally disappears (see figure 3), as it occurs for the AITG. The toroidal number of this mode is $n=8$, hence GAM is excluded, since it has $n=0$.

In figure 5(a), the contribution of each species to the time derivative of the potential energy is shown, concerning the mode at $k_{y}=0.15$. The effect of the fast ions is strong and the growth rate is largely enhanced, leading this low- $k_{y}$ instability to be the most dominant mode of the system. Before analyzing the impact of the fast ions on TEM, it is relevant to characterize this instability which is now driven essentially by the fast particles whereas it was driven only by the thermal deuterium species in the system without fast ions (case A) and it has been identified as an AITG mode. Indeed, also in this case the instability is localized in the spectrum and presents a very wide structure along the parallel direction (see figure 5(b)), with an interchange-like parity. We can safely say that the mode represented by this high peak is structurally the same as the mode detected in the system without fast ions (case A) at the corresponding region of the spectrum.

The same analysis of figure 2(b) has been carried out also for the fast-ion AITG mode (though for sake of simplicity, the plot has not been reported here) and it revealed that the instability in question is mainly driven by the passing fast ions, though the trapped ones are also giving energy to the mode. To verify it, the potential energy exchanged by the fast ions has been integrated in the passing and trapped domains of the velocity space. It results that only the $20 \%$ of the total energy transferred is coming from the trapped fast ions.

This latter mode is driven by both the total thermodynamic pressure of the system and the fast ion pressure gradient, as figures 6 (a) and (b) demonstrate, respectively. Indeed, as one can clearly see, any modification in these two different physical parameters leads to an acute alteration of the growth rates in the low- $k_{y}$ region. For sake of clarity, it is important to underline the consistent dependence that the electron $\beta$ has with the total $\beta$ - and also with the all ionic $\beta$ - through the pressure ratios $p_{s} / p_{e}$, where $p_{s}$ relates to the $s$-species. Therefore, the total $\beta$ parameter is strictly linked to $\beta_{e}$.

The linear transition from the AITG to this latter mode could be likely considered as a modification of the instability driver, from the thermal ion temperature gradient, which is known to be the driver of the AITG, to the fast ion pressure gradient. Since the mode is still excited in the BAE gap of the Alfvénic spectrum, this latter mode could be identified as a fast-ion-pressuregradient-driven BAE. For sake of simplicity, in the remainder of the paper, we will refer to this instability as FI-BAE.

\subsection{Linear Impact of Fast Ions on Trapped Electron Mode}

In figure 1(a), the impact of the fast ions on the spectrum is also examined, in both EM and ES simulations (see also figure 3 ). With the exception for the low- $k_{y}$ region $\left(k_{y}<0.35\right)$, the growth rates are not strongly affected by the presence of fast ions. Slight enhancement as well as minor shift to the right can be seen. This result is in counter-trend with the most-studied ITG dominated case of fast ion stabilization. Fast ions reduce the linear growth rates and even the nonlinear heat fluxes in a ITG dominated 


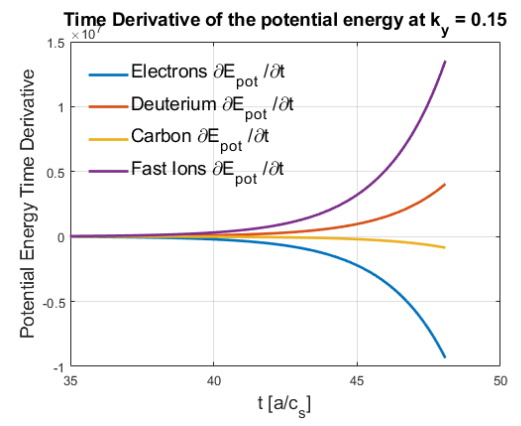

(a)

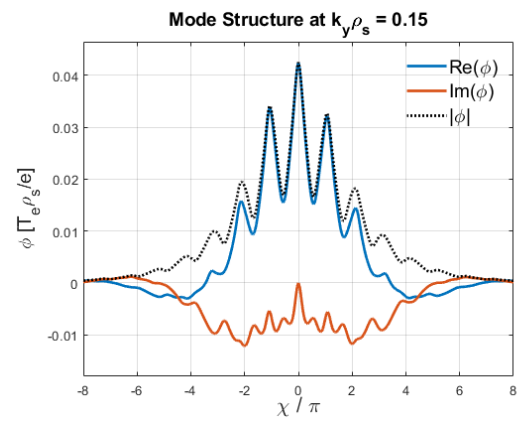

(b)

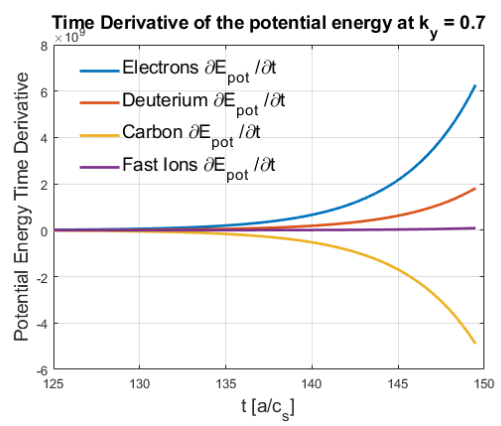

(c)

Figure 5: (a) Contribution of each species to the potential energy time derivative and (b) the electrostatic potential structure at $k_{y}=0.15$. The variable $\chi$ employed in the abscissa in (b) represents the toroidal angle in radians. In (c) the contribution of each species to the potential energy is shown for $k_{y}=0.7$, where the TEM dominates.

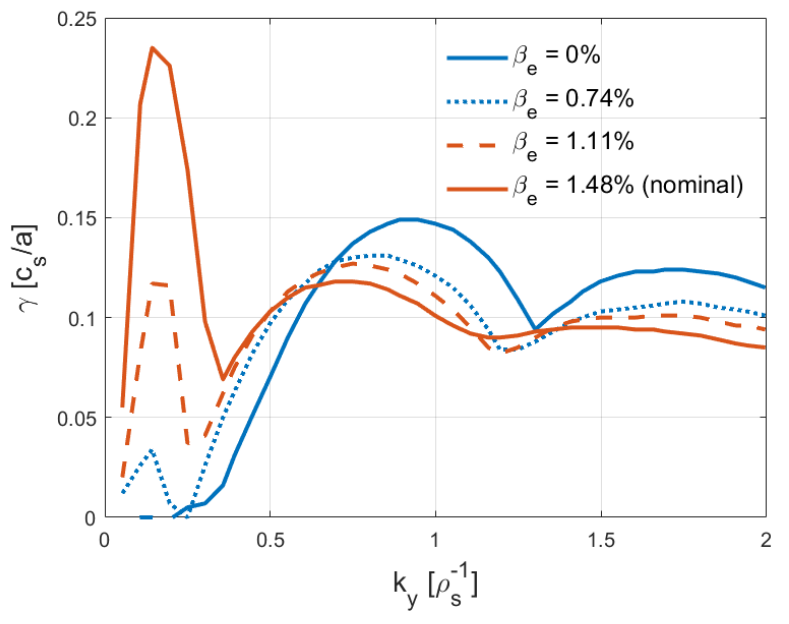

(a)

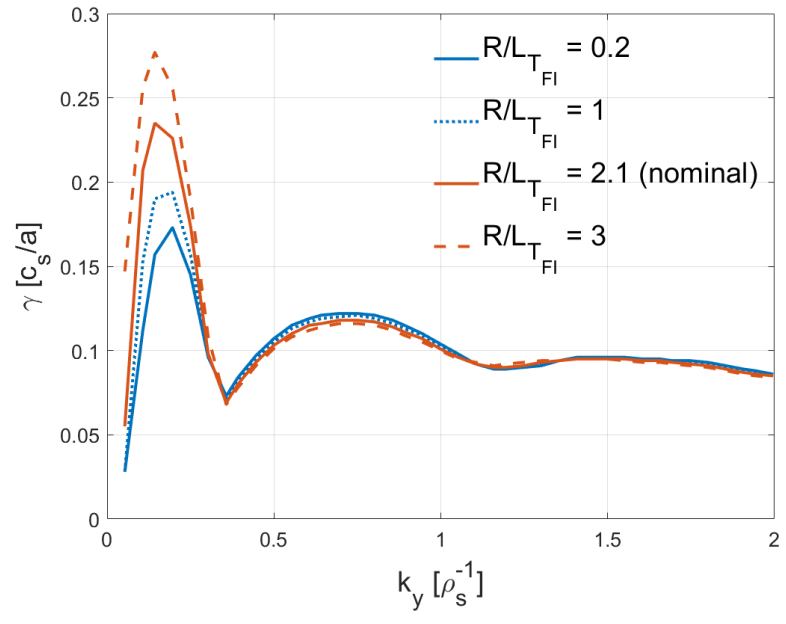

(b)

Figure 6: Linear growth rates for the scan over (a) the electron-beta $\beta_{e}$ and (b) the fast ion temperature gradient for the system with fast ions (case C) of JT-60U 48158 discharge.

JET Hybrid Scenario $[9,10]$. The same stabilization has been revealed also in the experiments to which the two previous references correspond [8,36,37]; in these discharges, a substantial reduction of turbulent heat transport has been observed concurrently with a significant fast ion population. It has been demonstrated that the stabilization occurs through a wave-particle resonance when the fast ion magneticdrift frequency overlaps the linear frequency of ITG mode in a favourable region of the phase space [6].

In contrast, the turbulent linear growth rate of TEM, that is the bulk-instability of this JT-60U shot, is not strongly affected by the fast ion presence, both in EM and ES systems. This can be seen in Figures 1 and 3 , where only weak effects occur when the fast ions are introduced in the simulations. Additionally, checking the fast particle contribution to the time derivative of the potential energy at $k_{y}=0.7$, no significant effect has been noted (see figure 5(c)). Moreover, no sign of influence on the TEM linear growth rates has been found when the fast ion pressure gradient varies in a wide range of values, see figure $6(\mathrm{~b})$. Nonetheless, the lack of linear resonant effect between fast ion and TEM could be also related to the fact that in this analysis a realistic NBI set of fast-ion parameters has been employed, i.e. $R / L_{T_{\text {fast }}} \ll R / L_{n_{\text {fast }}}$. Indeed, it was recently observed by means of GENE numerical simulations for a JET ITG-dominated case that, for resonance between fast-ion magnetic drift and TEM linear frequency to clearly occur, a ICRH setup is required $\left(R / L_{n_{\text {fast }}} \ll R / L_{T_{\text {fast }}}\right)[6,38]$. Furthermore, also $T_{\text {fast }}$ is shown to have an impact on the ITG- 
stabilization [6], but here has not been taken into account.

Summarizing, the effects of the fast ions on the TEM in this JT-60U hybrid scenario looks negligible, since no strong impact of the TEM linear growth rate has been appreciated. This has been analyzed for the first time with a gyrokinetic electromagnetic code such as GENE. However, further studies will be dedicated to analyze the impact of different $R / L_{T_{\text {fast }}} / R / L_{n_{\text {fast }}}$ and $T_{\text {fast }}$ on the TEM.

\section{Nonlinear Simulations}

In this section, the nonlinear regime simulations with GENE of JT-60U discharge \#48158 are examined. Firstly, the standard parameter case (case C) has been analysed. After realizing that the simulation setup needed to be modified to untangle the effects of AITG/FI-BAE modes on the transport, the thermal input parameters have been decreased within the experimental error bars and the fast ion pressure reduced to avoid the destabilisation of the AITG/FIBAE modes (cases F and G). Thus, it has been possible to study the nonlinear impact of the fast ions on the TEM.

\subsection{First Nonlinear Analyses of JT-60U \#48158 Discharge}

The first nonlinear simulations performed with GENE of JT-60U discharge are described in this subsection. The nominal parameter setup (case C) has been employed. In figure $7(\mathrm{~b})$, the scan of the thermal ion electrostatic heat diffusivity $\chi$ over $\beta_{e}$ for two different fast ion pressure gradients (cases D and E) is shown. It is to be noted that electromagnetic heat fluxes computed by the GENE code appear negligible with respect to the electrostatic ones, therefore they are not taken into account in the following. For both fastion pressure configurations a threshold value around $\beta_{e} \simeq 1.3 \%$, which lies within the range of the local experimental electron beta (valued as $\beta_{e}=1.48 \%$ ), is clearly visible. Beyond this value, the heat diffusivity of the thermal deuterium takes off, overestimating the experimental power balance diffusivity $\left(\chi_{i} \approx 1 \mathrm{~m}^{2} / \mathrm{s}\right)$ by more than 10 times at nominal $\beta_{e}$. Given the linear $\beta_{e}$ scan in figure $7(\mathrm{a})$ for certain $k_{y}$ - related to the region in which AITG/FI-BAE is excited - we can safely say that the transport increase is induced by the destabilization of the FI-BAE mode, which is driven by the plasma total pressure. As a matter of fact, in figure 8 , the electrostatic heat flux spectra of the same $\beta_{e}$ scan are compared, for the case in which the fast ion pressure gradient is lowered by $80 \%$ from the nominal value (case E). The spectra reveal that, after the threshold is exceeded, the heat fluxes peak at $k_{y} \simeq 0.2$ that is extremely close to the length scale at which the FI-BAE is linearly destabilized. On the other hand, when the FI-BAE mode is stable or slightly excited, i.e. for the cases below $\beta_{e}=1.3$, the transport values are almost negligible and the spectra do not peak in the low- $k_{y}$ region. This was expected, since already looking at figure 1 , the solid profiles, with and without fast ions, labelled as standard cases (cases A and C), exhibit the destabilization of AITG/FI-BAE modes in the low- $k_{y}$ region of the spectrum.

It is relevant to say that the fast ion heat transport is enhanced by the total pressure of the system: it goes from $Q_{E S}^{\text {fast }} \simeq 1 \mathrm{~kW} / \mathrm{m}^{2}$ for $\beta_{e}<1.3 \%$ to $Q_{E S}^{f a s t} \simeq 10 \mathrm{~kW} / \mathrm{m}^{2}$ when the threshold is exceeded; and so the electromagnetic part of the fast-ion heat flux $Q_{E M}^{\text {fast }}$ does, increasing from a negligible value (around $\left.Q_{E M}^{f a s t} \simeq 0.3 \mathrm{~kW} / \mathrm{m}^{2}\right)$ up to two orders of magnitude higher values $\left(Q_{E M}^{f a s t} \simeq 32 \mathrm{~kW} / \mathrm{m}^{2}\right)$. This result goes in the same direction with respect to the increase of the linear growth rate when the total pressure of the system is enhanced. Actually, though the thermal ion heat flux increment reflects the linear growth rate one, the ratios among the different steps of the nonlinear $\beta$-scan are greater than the corresponding linear ones. This may indicate a nonlinear synergy between FI-BAE and some other modes, which boosts the transport up more than the linear saturation mechanism (see figures 6(a) and 8 for comparison).

Nevertheless, the fast ion heat fluxes are even more increased if the fast ion density gradient is considered at $50 \%$ of the nominal value (case D), instead of the $25 \%$ (case E) of the standard case, nonetheless for sake of simplicity we do not report a figure for that. In figure 7 , the striking observation is that, when $\beta_{e}<1.3 \%$, the two cases (cases $\mathrm{D}$ and $\mathrm{E}$ ) have very similar thermal ion heat diffusivities. This consideration demonstrates that the increasing of the thermal ion heat flux is mainly due to the FI-BAE instability, which is likely to dominate beyond the threshold. In fact, the nonlinear synergy between the fast-ion driven instabilities and the unstable thermal modes is enhanced by the electromagnetic effects, as figure 7(b) demonstrates. In addition, if the fast ion pressure gradient is increased, the ion heat diffusivity grows steeper, likely due to the enhancement of the instability driver.

In light of such nonlinear results obtained for the nominal input parameters, a change of the simulation setup was established to be necessary. This change is motivated by the fact that at the nominal $\beta_{e}$ value, the possible strong nonlinear synergy between AITG/FI-BAE and TEM leads to an heat flux value much larger than that observed in the experiments and that the aformentioned FI-BAE instability has not been detected experimentally in the JT-60U discharge 


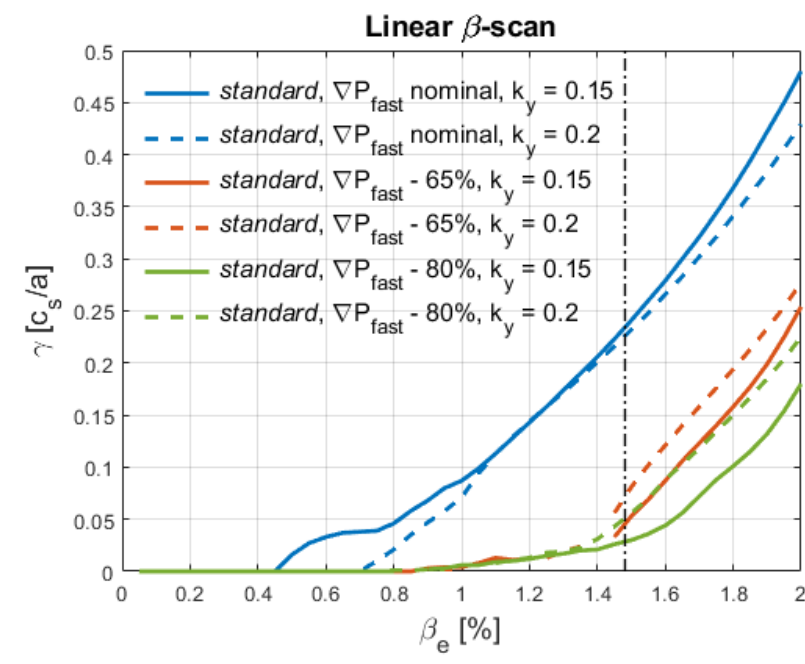

(a)

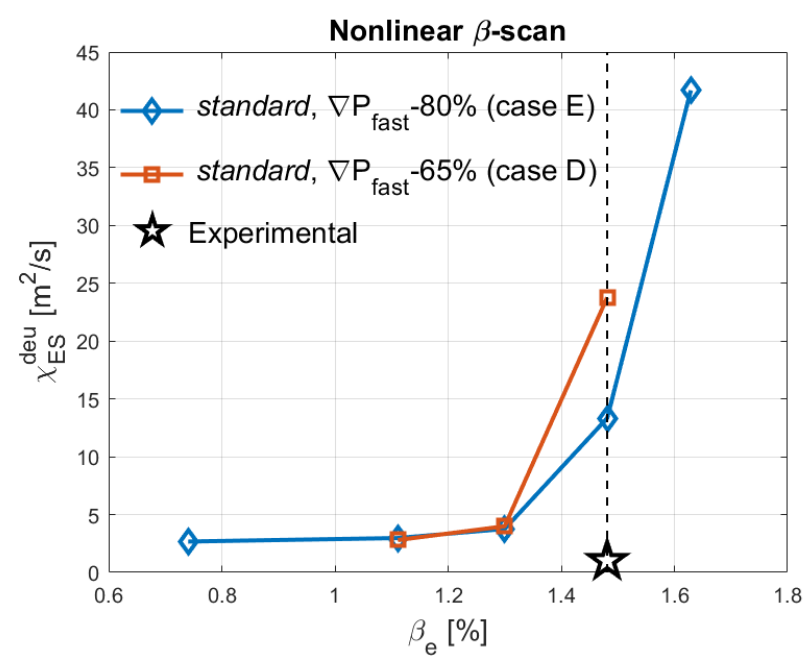

(b)

Figure 7: In (a), the linear $\beta$-scan for different $k_{y}$ and three different cases of fast ion pressure gradient is shown. The blue profiles refer to case C, the red to case D and the green to case E. In (b), Deuterium electrostatic heat diffusivity for two different fast ion pressure gradient cases - the same of (a) - is shown over a electron $\beta$-scan. It is important to stress that the scan over electron $\beta$ has been performed with the standard case input parameters, in order to have the FI-BAE linearly excited. The black vertical dashed line represents the nominal $\beta_{e}$ of the standard cases, whereas the black star the experimental heat diffusivity [18].

\#48158. In this sense, a global simulation - which allows for the radial redistribution of the fast particles and the consequent modification of the pressure profiles - would be useful to evaluate the stability of the FIBAE in this particular JT-60U scenario. Nevertheless, this is out of the scope of the present work. In addition, to clearly isolate the direct effect of fast ions on TEM turbulence, which is the main goal of this paper, the AITG/FI-BAE instabilities need to be stabilised.

\subsection{Explanation of modified setup}

As it has been demonstrated in the previous subsection, in order to analyse the interaction among fast ions and TEM and avoid unrealistic high transport levels, the AITG/FI-BAE needs to be stabilized. In addition, it is to be noted that the aforementioned FI-BAE instability has not been detected in the JT60U discharge \#48158. Therefore, this situation is similar to that encountered at JET for ITG dominated hybrid regimes $[9,10]$, where BAE modes had to be stabilized in order to reconcile power balance and GENE calculated heat fluxes.

We proceed therefore in a similar way by performing simulations within the experimental uncertainties (assumed to be of the order of 20\%). In the following, we will refer to the nominal case as the standard one, whereas the modified case is the one in which the input parameters for the thermal ions are altered con-

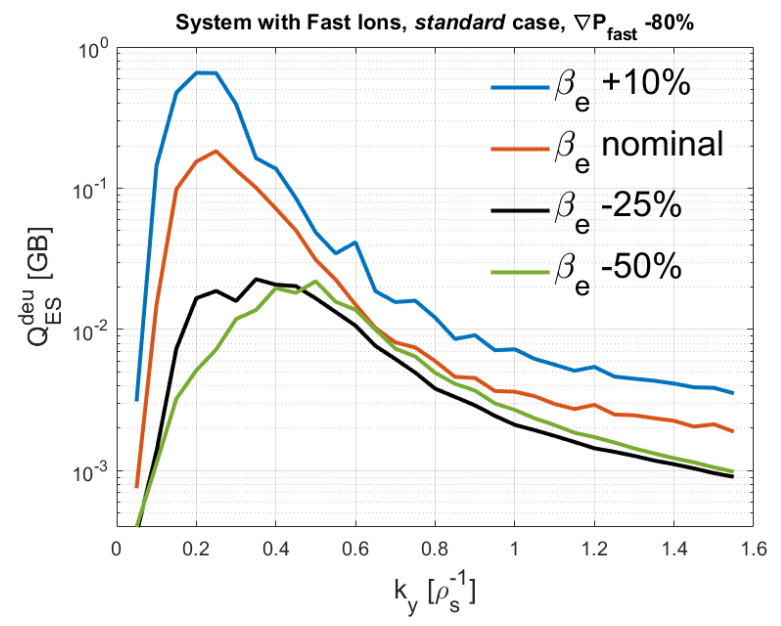

Figure 8: Deuterium electrostatic heat flux spectra are shown in a scan over the $\beta_{e}$, based on case $\mathrm{E}$ for which the fast ion pressure gradient is lowered by $80 \%$. It is important to stress that the scan over electron beta has been performed with the standard case input parameters, in order to have the FI-BAE linearly excited at the nominal $\beta_{e}$.

sistently within the error bars. All the modifications from the standard setup are listed in table 2 . The main alterations concern the thermal ion temperature gradient, that has been decreased below the threshold for the destabilization of the AITG, the thermal ion 


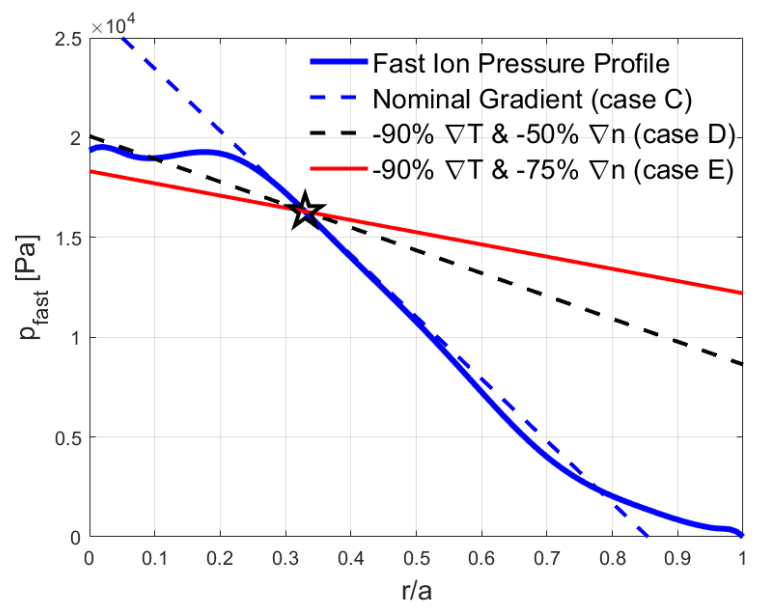

Figure 9: Fast ion pressure profile. Various pressure gradients used in simulations are also shown. Black star represents the radial position at which the simulations are performed.

density, that has been lowered, and finally the electron $\beta$, that has been decreased. A special mention must be done for the input parameters regarding fast ion species: a significant reduction of the fast ion pressure gradient has been considered in this paper. Indeed, as it is explained in the previous subsection, if the nominal pressure profile is employed in nonlinear simulations (case $\mathrm{C}$ ), it results in fluxes widely larger than experimental observations and then unreliable (up to more than 100 times larger). This is likely due to FIBAE instability, which enhances the transport of all the species. Consequently, a stronger reduction of fast ion temperature gradient (decreased by $50 \%$ or $90 \%$ from the nominal value) than density gradient (lowered by 50 or $75 \%$ from the nominal value) has been considered, in order to get closer to NBI-like setup, where usually $R / L_{T_{\text {fast }}} \ll R / L_{n_{\text {fast }}}$. For sake of clarity, case $\mathrm{F}$ presents a reduction by $60 \%$ for the temperature gradient and $50 \%$ for the density gradient, whereas case $\mathrm{G}$ by $90 \%$ for the temperature gradient and $75 \%$ for the density gradient.

In figure 9, the fast ion pressure profile is shown as it was originally computed. The black star represents the normalized radial position of the simulations performed, whereas the different straight lines describe the various cases taken into account and listed in table 2. In figure 1 these modifications have been applied as input parameters in simulations and the linear results are represented by the dotted profiles. It is to be noted that, in the modified case (case G), the fast ion pressure gradient is represented by the red straight line in figure 9, thus critical decreases of $90 \%$ for the temperature and $75 \%$ for the density gradient have been applied.

In figure 1, we can also appreciate the comparison among the linear spectra of different cases of the simulation without fast ions, specifically the standard (case A), the modified (case B) and the case in which, starting from the standard input parameters (case A), the thermal deuterium temperature gradient was decreased down to $R / L_{T_{i}}=5$. The choice of the latter value is based on the scan over the temperature gradient of the thermal ion species that could be seen in figure 4 . In fact, the modified case uses $R / L_{T_{i}}=6.21$ that is already below the AITG threshold, nonetheless also other modifications have been applied to the thermal species parameters (see table 2). Indeed, it is to be noted that in the modified case (case B) the thermal ion density is decreased in order to stabilize the AITG, but this change also alters the impurity density, for fulfilling the quasineutrality relation. Hence, as we can appreciate in figure 1, the modifications affect also the carbon-ITG, whose growth rate in the high- $k_{y}$ region is enhanced, as well as the TEM. This effect is also visible in the black dotted profile. Therefore, the enhancement of the TEM growth rate is totally due to the decrease of the deuterium temperature gradient below the AITG threshold, since the profiles of the modified and the case in which just the deuterium $R / L_{T_{i}}$ is lowered fit almost perfectly around the TEM-dominated region $\left(0.3<k_{y}<1.2\right)$. We can draw the conclusion that, when fast ions are not included as a kinetic species, the dominant instability is not a pure TEM, but it is affected by the thermal deuterium temperature gradient, namely it is stabilized. This result could be explained by the fact that the destabilizing or stabilizing regions in the system velocity space could be altered when the thermal deuterium pressure profile is modified. Therefore, resonances between particles and instability waves are moving in the velocity space when the thermal deuterium pressure profile is altered. From this latter consideration, since TEM is well known to be excited by the resonance with trapped electron motions, we could draw the conclusion that TEM resonant region is moving to destabilized phase space range when thermal ion temperature gradient is decreased.

\subsection{Nonlinear Impact of Fast Ions on Trapped Electron Mode}

In order to disentangle all the possible effects on the interaction between TEM and fast ions, as it has been done linearly, the input parameters for this subsection are those of the modified cases (cases F and G). In fact, the AITG/FI-BAE modes are now linearly stable and the system can be considered definitely TEMdominated, as figure 1 shows. However, the carbonITG, that is linearly excited at $k_{y} \simeq 1.5$, is still unstable. Therefore, the numerical domain box has 
been chosen such in a way that the $k_{y}>1.6$ region is not retained in the simulations for the avoidance of carbon-ITG effects on the system.

In figures 10 the electrostatic heat fluxes and spectra for the thermal ion and electron species are compared between the modified cases without (case B) and with fast ions (case F and G), in (a) and in (b) respectively. This comparison sheds light on the transport induced by the TEM, which is known to develop at the ion scale [39]. It can be observed that both the time trace and the spectra are very close between their respective cases with and without fast ions. The time trace underlines a strong correspondence even concerning the saturation phase, especially for the thermal deuterium species. Furthermore, the two-point scan over the fast ion pressure gradient corroborates the result. As a matter of fact, no substantial difference between case $\mathrm{F}$ and case G can be appreciated. Therefore, this result confirms what has been said for the linear analysis, i.e. the fast ions are not affecting substantially the TEM-induced transport in this JT-60U discharge. As it has already been observed before, this result is in counter-trend with respect to the ITG-dominated cases, which showed a relative strong stabilization due to fast ion presence in a wide range of fast ion physical parameters such as $T_{\text {fast }}$ and $R / L_{T_{\text {fast }}} / R / L_{n_{\text {fast }}}[9$, 10].

For sake of simplicity, in figure 10(a), the comparison for the carbon species is not reported; however, the electrostatic heat fluxes only differ for less than $5 \%$ between the cases with fast ions and the case without.

A possible explanation of this nonlinear behaviour could be linked to the role of zonal flows in the saturation process. The ITG-dominated case saturation process through zonal flows is well-known [40]. On the other side, as it has been suggested, the zonal flows are not actively involved in the nonlinear saturation mechanism of electron temperature gradient driven TEM [41]. According to [42], the role of zonal flow was found to be negligible when $R / L_{T_{e}}>$ $R / L_{n_{e}}$, that is the case of this JT-60U 48158 discharge (see table 1). The impact of the zonal component on the saturation mechanism has been analysed by computing the ratio between the zonal flow shearing rate, $\gamma_{E x B, z o n a l}$, and the maximum of the linear growth rate, $\gamma_{\max }$ [43]. This analysis is still valid for the $k_{y}<2$ region, as reference [44] demonstrates. Nevertheless, it is to be noted that if the impact of the zonal flow on the large- $k_{y}$ spectrum region is considered, then it is important to use another approach detailed in Ref. [44]. Therefore, in our case, since the interesting region is localized at $k_{y}<1$, the comparison of the ratios $\gamma_{E x B \text {, zonal }} / \gamma_{\max }$ is more convenient. For sake of clarity, the zonal shearing rate is defined as $\gamma_{E x B, z o n a l}=\left|k_{x}^{2} \phi\left(k_{x}, 0\right)\right|$. The results of this analysis are reported in table 3 . It is important to note that the modified cases, both with and without fast ions, have a comparable value of the ratio $\gamma_{E x B, \text { zonal }} / \gamma_{\max }$ and also a less significant zonal flow impact than standard cases. This statement could imply that, since the modified cases present only the linear destabilization of TEM, zonal flow components do not substantially affect turbulent transport and thus fast ions cannot interact through this channel. Nevertheless, it must be highlighted that, in order to properly compute the radial structure of zonal flows, global simulations should be employed instead of the local approximation here used. However, global simulations are out of the scope of this work, thus future analyses will be dedicated to this topic.

On the other hand, in the standard cases, also AITG (and FI-BAE) modes are destabilized. Therefore, we are suggesting here that probably there is a transfer of energy from the AITG (and FI-BAE) modes to the zonal flows in their saturation process. However, also this is out of the scope of the present work, nonetheless it will be analyzed in the near future.

Furthermore, the fast ion heat flux, which is not represented directly here, was found to be negligible in the experiment. Therefore, no fast ion diffusivity was assumed in the OMFC Monte Carlo calculations performed to compute the fast ion profiles. And in fact, the nonlinear simulations for the modified cases (cases $F$ and $G$ ) give insignificant fast ion transport. It is relevant to notice that also a rough agreement between the experimental ion diffusivity and the GENE simulated diffusion coefficient of the modified case has been achieved. As it could be easily derived from reference [18], at $t=27 \mathrm{~s}$ the thermal ion diffusion coefficient is estimated as $\chi_{i} \simeq$ $1 \mathrm{~m}^{2} / \mathrm{s}$, whereas our computed result is $\chi_{i}=2.4$ $\mathrm{m}^{2} / \mathrm{s}$. The difference between the two values should be evaluated in the context of the large experimental uncertainties on $q$-profile, very low value of magnetic shear and unlikely realistic high impurity density in the core. Hence, we can conclude that the rough agreement achieved is noteworthy. However, before a conclusive and definitive experimental interpretation can be offered, global analyses would be extremely useful, since features such as the redistribution of fast ions and modification of pressure profiles are not taken into account in the local approximation. These studies are left for future efforts.

\section{Conclusions}

In conclusion, we have studied the impact of fast ions on the TEM microinstability arising in a JT- 


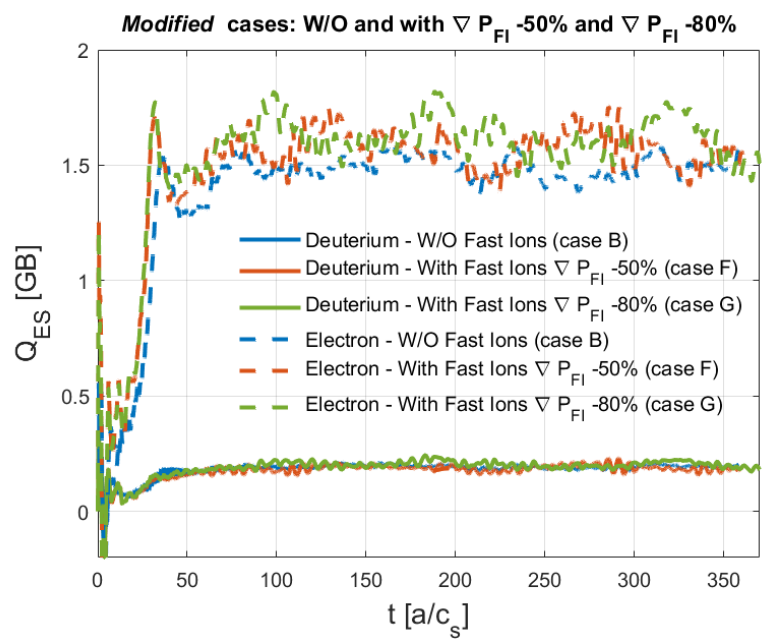

(a)

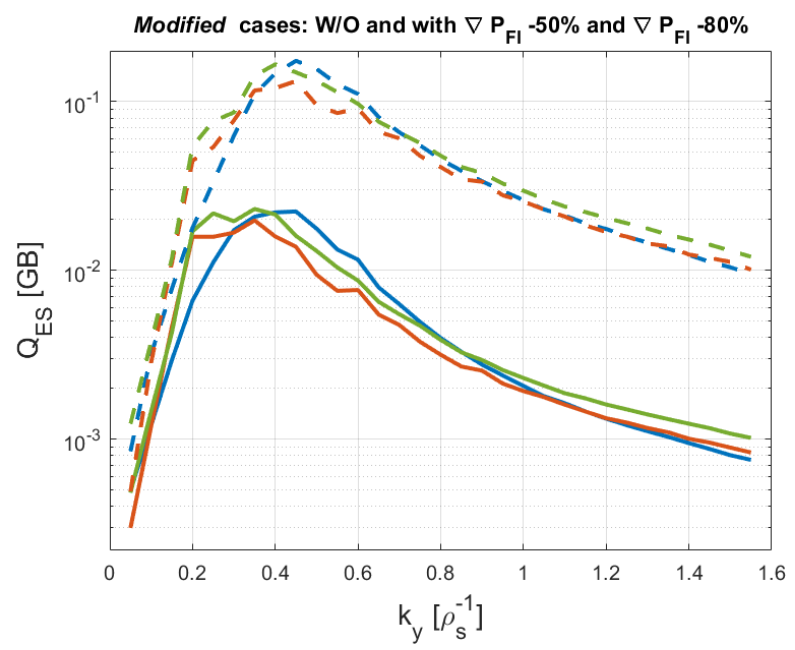

(b)

Figure 10: (a) Time trace comparison between the electrostatic heat fluxes with and without fast ions for the modified cases of electron and thermal deuterium species. The spectra of the main thermal ion species and the electron are compared between the system with and without fast ions in (b). The two plots refer to the same legend.

Table 3: Zonal flow impact obtained for different cases. It is to be noted that the fast ion pressure gradient is lowered by $\simeq 50 \%$ (case $\mathrm{F}), \simeq 65 \%$ (case D) and $\simeq 80 \%$ (cases $\mathrm{E}$ and $\mathrm{G}$ ) for the cases in which fast ions are included. The identification of each case is referred to table 2.

\begin{tabular}{ccc}
\hline & Case & $\gamma_{E \times B, \text { zonal }} / \gamma_{\max }$ \\
\hline $\mathrm{A}$ & standard $-\mathrm{W} / \mathrm{O}$ Fast Ions & 1.87 \\
$\mathrm{~B}$ & modified $-\mathrm{W} / \mathrm{O}$ Fast Ions & 1.23 \\
$\mathrm{D}$ & standard $-65 \% \nabla P_{\text {fast }}-$ With Fast Ions & 3.29 \\
$\mathrm{E}$ & standard $-80 \% \nabla P_{\text {fast }}-$ With Fast Ions & 2.92 \\
$\mathrm{~F}$ & modified $-50 \% \nabla P_{\text {fast }}$ - With Fast Ions & 1.03 \\
$\mathrm{G}$ & modified $-80 \% \nabla P_{\text {fast }}-$ With Fast Ions & 1.04 \\
\hline
\end{tabular}

$60 \mathrm{U}$ hybrid scenario. The analyses were carried out by means of the gyrokinetic GENE code, which was used in a local approach. It was shown that fast ions only marginally affect the TEM linear growth rate. The study is then extended to the nonlinear regime, which demonstrate that fast ions, as long as AITG/FI-BAE modes are not destabilized, do not affect the TEM-induced turbulent transport for the case under investigation. A possible explanation for this lack of interplay might be attributed to zonal flows. In fact, the nonlinear saturation mechanism for electron temperature driven TEM has been demonstrated not to be the zonal flow generation, which in contrast plays an important role in the ITGdriven transport reduction. Indeed, ITG turbulence reduction is known to be enhanced by the fast ions, both experimentally and numerically in a wide range of fast ion physical parameters. This effect, however, is established up to the destabilization of AITG - and its transition to a more dominant fast-ion-driven instability when fast ions are included consistently in the simulation setup. These instabilities were found to be unstable in our GENE input parameter range, but was not detected by the experimental diagnostics. Therefore, we modified the parameter inputs within the experimental uncertainties in order to stabilize these low- $k_{y}$ instabilities and thus to untangle their effect on turbulent transport. In such a manner, it was possible to analyse the impact of fast ions on TEM-induced turbulence in a parameter regime which yields transport fluxes being in reasonable agreement with the experimental heat fluxes measurements. Since, the validity of the local approach employed was cautiously guaranteed by dedicated analyses, the simulations reported in this paper imply good qualitative indications about the impact of fast ions on TEM. Indeed, global simulations would be useful for quantitative comparisons with experimental 
measurements and also to properly compute the radial structure of zonal flows, which might play an important role in the total underlying process. Nevertheless, such an equivalent global tool including all the features employed - i.e. mainly electromagnetic effects, multi ions, kinetic electrons, real geometry - is extremely demanding computationally.

The results obtained in this paper have strong consequences on how plasma turbulence suppression can be controlled and optimized. As an example, whereas optimization of JET plasmas towards high confinement regimes relies on the increase of $\beta$ and fast ion through the NBI heating, in other tokamaks such path could be not effective due to differences in the underlying turbulence. Therefore, it is essential to study such mechanisms in the specific plasma configurations for the different tokamaks. This is essential for tokamak devices such as JT-60SA, where the NBI will mostly heat the electrons due to its high voltage, or ITER, where alpha particles will also heat the electrons.

Eventually, in order to come to a final and definitive answer about the impact of fast ions on microturbulence, a self-consistent modelling which includes heating codes, small-scale turbulence and fast-ion-driven instability effects is required. This is especially important in order to determine a coherent thermal and fast ion transport. This topic will be specifically studied in future analyses.

\section{Acknowledgments}

This work has been carried out within the framework of the EUROfusion Consortium and has received funding from the Euratom research and training programme 2014-2018 and 2019-2020 under grant agreement No 633053. The views and opinions express herein do not necessarily reflect those of the European Commission.

Simulations were performed both on IRENE Joliot-Curie HPC, in the framework of the PRACE project IONFAST, and CINECA Marconi HPC within the project GENE4EP.

The authors would like to acknowledge G.L. Falchetto and X. Garbet for stimulating discussions and fruitful suggestions. A. Bierwage is especially thanked for his passionate warnings about the validity of the ballooning representation in the local approximation in gyrokinetic codes which allowed us to deepen the analyses made.

\section{Appendix A. Study on the Validity of Local Approach in GENE Linear Simulations of JT60U \#48158 Discharge}

In this appendix, we show the validity of the local approximation employed in the analysis of JT-60U \#48158 discharge, checking the linear mode structures in the real space domain.

For sake of simplicity, only three different linear mode structures - corresponding to the three regions identified in section 3 - are shown in figure A1. The regions are representative of the three different modes excited in the spectra, i.e. AITG/FI-BAE, TEM and Carbon-ITG. The panels (a), (b) and (c) exhibit the electrostatic potential mode structures in the real domain, obtained by Fourier transforming the corresponding mode structures in the ballooning space. Indeed, panels (d), (e) and (f) represent the mode structures in the ballooning representation. Thus, the convenient coordinate $n\left(q_{0}-q(r)\right)$, where $n$ and $q_{0}$ are respectively the toroidal wavenumber and the value of the safety factor at the selected flux tube radial position (see table 1), has been exploited in the abscissa for underlying the rational surfaces in real domain.

In figure A1(a), one can appreciate that the mode at $k_{y}=0.15$ is radially localized, since its dominant peak is sitting at the rational surface $n\left(q_{0}-q(r)\right)$, whereas the peaks at the other integer values of $n\left(q_{0}-q(r)\right)$ coordinate are relatively small. Therefore, the local approach is valid for the dominant modes (AITG/FI-BAE) excited in this region. Regarding the second region, i.e. $0.35<k_{y}<1.2$, where TEMs dominate, a coupling between the neighbouring rational surfaces occurs, as one can appreciate in panel (b) of figure A1. In this case the principle peaks are localized at $n\left(q_{0}-q(r)\right)= \pm 0.5$, which means that the mode has a finite value of $k_{\|}=(n q-$ $m) / q R$. Nonetheless, the validity of the local approach is ensured by the small ratio between the dominant peaks and its side-bands, which do not overcome $20 \%$. Finally, for what concerns the rightmost region of the spectrum, i.e. $k_{y}>1.2$, dominated by the Carbon-ITG, the ballooning representation in the local approach is intrinsically valid, since the safety factor and plasma parameter variations from one rational surface to the following one are small (limit for $n \gg 1$ ), i.e. the translational invariance is fulfilled. However, for completeness the structure of the Carbon-ITG in $n\left(q_{0}-q(r)\right)$ domain is displayed in figure $\mathrm{A} 1(\mathrm{c})$. 


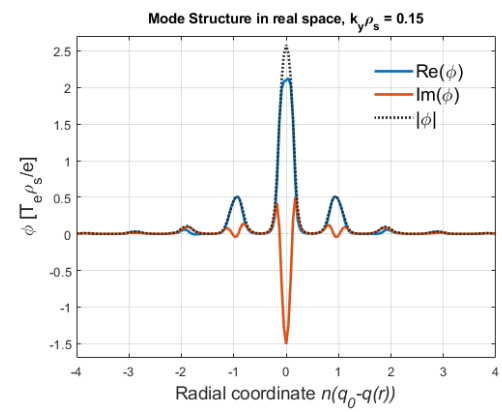

(a)

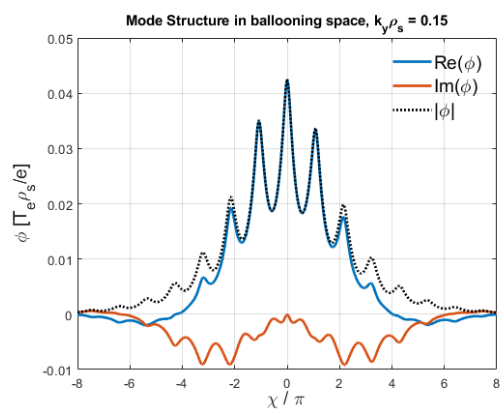

(d)

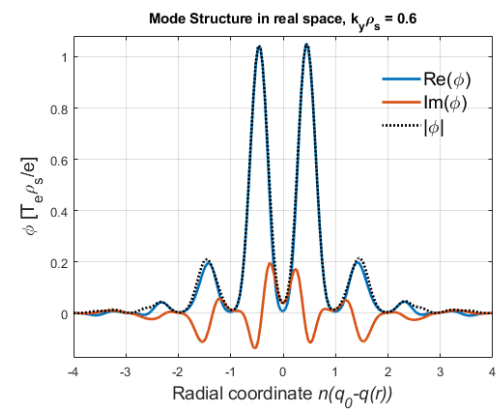

(b)

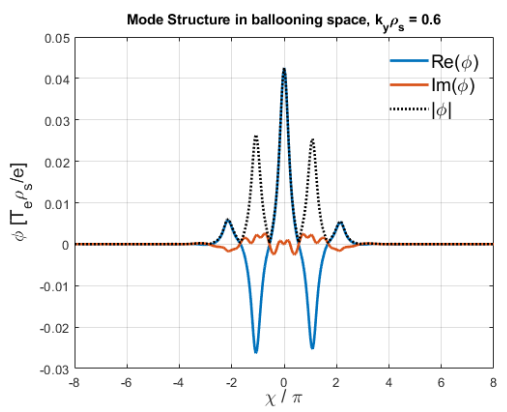

(e)

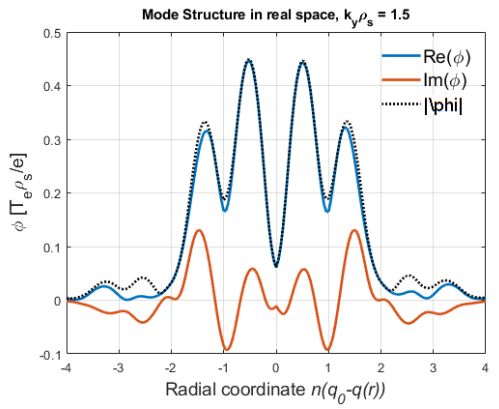

(c)

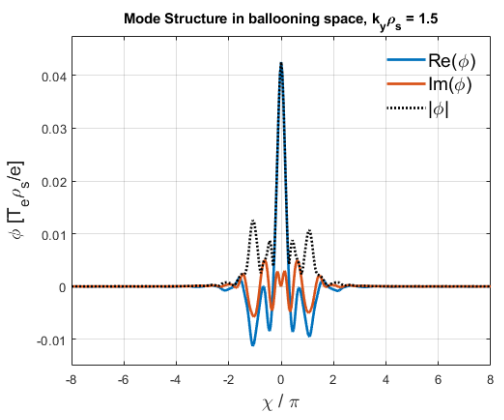

(f)

Figure A1: In (a), (b) and (c) the electrostatic potentials $\phi$ in the real domain for $k_{y} \rho_{s}=0.15, k_{y} \rho_{s}=0.6$ and $k_{y} \rho_{s}=1.5$ are respectively shown. In (d), (e) and (f) the corresponding structures in the ballooning domain are also reported. It is to be noted that these structures refer to case $\mathrm{C}$.

\section{References}

[1] D. Zarzoso, D. del Castillo-Negrete, D.F. Escande, Y. Sarazin, X. Garbet, V. Grandgirard, C. Passeron, G. Latu, and S. Benkadda. Particle transport due to energetic-particle-driven geodesic acoustic modes. Nuclear Fusion, 58(10):106030, 2018.

[2] L. Chen and F. Zonca. Physics of alfvén waves and energetic particles in burning plasmas. Reviews of Modern Physics, 88(1):015008, 2016.

[3] P. Lauber. Super-thermal particles in hot plasmas - kinetic models, numerical solution strategies, and comparison to tokamak experiments. Physics Reports, 533(2):33-68, 2013.

[4] G. Tardini, J. Hobirk, V.G. Igochine, C.F. Maggi, P. Martin, D. McCune, A.G. Peeters, A.C.C. Sips, A. Stabler, J. Stober, et al. Thermal ions dilution and itg suppression in asdex upgrade ion itbs. Nuclear fusion, 47(4):280, 2007.

[5] M. Romanelli, A. Zocco, F. Crisanti, JET-EFDA Contributors, et al. Fast ion stabilization of the ion temperature gradient driven modes in the joint european torus hybridscenario plasmas: a trigger mechanism for internal transport barrier formation. Plasma Physics and Controlled Fusion, 52(4):045007, 2010.

[6] A. Di Siena, T. Görler, H. Doerk, E. Poli, and R. Bilato. Fast-ion stabilization of tokamak plasma turbulence. Nuclear Fusion, 58(5):054002, 2018.

[7] A. Di Siena, T. Görler, E. Poli, A. Bañon-Navarro, A. Biancalani, and F. Jenko. Electromagnetic turbulence suppression by energetic particle driven modes. Nuclear Fusion, 59(12):124001, 2019.

[8] J. Citrin, F. Jenko, P. Mantica, D. Told, C. Bourdelle,
J. Garcia, J.W. Haverkort, G.M.D. Hogeweij, T. Johnson, and M.J. Pueschel. Nonlinear stabilization of tokamak microturbulence by fast ions. Physical review letters, 111(15):155001, 2013.

[9] J. Citrin, J. Garcia, T. Görler, F. Jenko, P. Mantica, D. Told, C. Bourdelle, D.R. Hatch, G.M.D. Hogeweij, T. Johnson, et al. Electromagnetic stabilization of tokamak microturbulence in a high- $\beta$ regime. Plasma Physics and Controlled Fusion, 57(1):014032, 2014.

[10] J. Garcia, C. Challis, J. Citrin, H. Doerk, G. Giruzzi, T. Görler, F. Jenko, P. Maget, and JET Contributors. Key impact of finite-beta and fast ions in core and edge tokamak regions for the transition to advanced scenarios. Nuclear Fusion, 55(5):053007, 2015.

[11] H. Doerk, C. Challis, J. Citrin, J. Garcia, T. Görler, F. Jenko, and JET Contributors. Gyrokinetic study of turbulence suppression in a jet-ilw power scan. Plasma Physics and Controlled Fusion, 58(11):115005, 2016.

[12] G.J. Wilkie, A. Iantchenko, I.G. Abel, E. Highcock, I. Pusztai, and JET Contributors. First principles of modelling the stabilization of microturbulence by fast ions. Nuclear Fusion, 58(8):082024, 2018.

[13] H. Doerk, A. Bock, A. Di Siena, E. Fable, T. Görler, F. Jenko, J. Stober, et al. Turbulence in high-beta asdex upgrade advanced scenarios. Nuclear Fusion, 58(1):016044, 2017.

[14] J. Garcia, T. Görler, and F. Jenko. Isotope and fast ions turbulence suppression effects: Consequences for high- $\beta$ iter plasmas. Physics of Plasmas, 25(5):055902, 2018.

[15] D. Zarzoso, Y. Sarazin, X. Garbet, R. Dumont, A. Strugarek, J. Abiteboul, T. Cartier-Michaud, G. DifPradalier, Ph. Ghendrih, V. Grandgirard, et al. Physical Review Letters, 110(12):125002, 2013. 
[16] D Zarzoso, P Migliano, V Grandgirard, G Latu, and C Passeron. Nucl. Fusion, 57(072011):072011, 2017.

[17] G. Giruzzi, M. Yoshida, J.F. Artaud, Ö. Asztalos, E. Barbato, P. Bettini, A. Bierwage, A. Boboc, T. Bolzonella, S. Clement-Lorenzo, et al. Physics and operation oriented activities in preparation of the jt-60sa tokamak exploitation. Nuclear Fusion, 57(8):085001, 2017.

[18] N. Oyama, A. Isayama, G. Matsunaga, T. Suzuki, H. Takenaga, Y. Sakamoto, T. Nakano, Y. Kamada, S. Ide, et al. Long-pulse hybrid scenario development in jt-60u. Nuclear Fusion, 49(6):065026, 2009.

[19] J. Garcia, N. Hayashi, B. Baiocchi, G. Giruzzi, M. Honda, S. Ide, P. Maget, E. Narita, M. Schneider, H. Urano, et al. Physics comparison and modelling of the jet and jt$60 \mathrm{u}$ core and edge: towards jt-60sa predictions. Nuclear Fusion, 54(9):093010, 2014.

[20] F. Jenko, W. Dorland, M. Kotschenreuther, and B.N. Rogers. Electron temperature gradient driven turbulence. Physics of Plasmas, 7(5):1904-1910, 2000.

[21] G.T.A. Huysmans, J.P. Goedbloed, and W. Kerner. Cp90 conf. on computational physics, 1991.

[22] J.F. Artaud, V. Basiuk, F. Imbeaux, M. Schneider, J. Garcia, G. Giruzzi, P. Huynh, T. Aniel, F. Albajar, J.M. Ané, et al. The cronos suite of codes for integrated tokamak modelling. Nuclear Fusion, 50(4):043001, 2010.

[23] K Tani, M Azumi, H Kishimoto, and S Tamura. Effect of toroidal field ripple on fast ion behavior in a tokamak. Journal of the Physical Society of Japan, 50(5):17261737,1981

[24] FL Hinton and Richard D Hazeltine. Theory of plasma transport in toroidal confinement systems. reviews of Modern Physics, 48(2):239, 1976.

[25] A. Bañon Navarro, P. Morel, M. Albrecht-Marc, D. Carati, F. Merz, T. Görler, and F. Jenko. Free energy balance in gyrokinetic turbulence. Physics of plasmas, 18(9):092303, 2011.

[26] J. Weiland and A. Hirose. Electromagnetic and kinetic effects on the ion temperature gradient mode. Nuclear Fusion, 32(1):151, 1992.

[27] A. Hirose. On finite $\beta$ stabilization of the toroidal ion temperature gradient mode. Physics of Plasmas, $7(2): 433-436,2000$

[28] F. Zonca, L. Chen, J.Q. Dong, and R.A. Santoro. Existence of ion temperature gradient driven shear alfvén instabilities in tokamaks. Physics of Plasmas, 6(5):19171924,1999

[29] J. Dong, L. Chen, and F. Zonca. Study of kinetic shear alfvén modes driven by ion temperature gradient in tokamak plasmas. Nuclear Fusion, 39(8):1041, 1999.

[30] G.L. Falchetto, J. Vaclavik, and L. Villard. Globalgyrokinetic study of finite $\beta$ effects on linear microinstabilities. Physics of Plasmas, 10(5):1424-1436, 2003.

[31] W. Chen, R.R. Ma, Y.Y. Li, Z.B. Shi, H.R. Du, M. Jiang, L.M. Yu, B.S. Yuan, Y.G. Li, Z.C. Yang, et al. Alfvénic ion temperature gradient activities in a weak magnetic shear plasma. EPL (Europhysics Letters), 116(4):45003, 2017.

[32] C.Z. Cheng. Kinetic theory of collisionless ballooning modes. The Physics of Fluids, 25(6):1020-1026, 1982.

[33] W.W. Heidbrink, E. Ruskov, E.M. Carolipio, J. Fang, M.A. Van Zeeland, and R.A. James. What is the "beta-induced alfvén eigenmode?". Physics of Plasmas, 6(4):1147-1161, 1999.

[34] F. Zonca, L. Chen, and R.A. Santoro. Kinetic theory of lowfrequency alfvén modes in tokamaks. Plasma physics and controlled fusion, 38(11):2011, 1996.

[35] D. Zarzoso, X. Garbet, Y. Sarazin, R. Dumont, and V. Grandgirard. Physics of Plasmas, 19(2):022102022102, 2012.
[36] P. Mantica, D. Strintzi, T. Tala, C. Giroud, T. Johnson, H. Leggate, E. Lerche, T. Loarer, A.G. Peeters, A. Salmi, et al. Experimental study of the ion critical-gradient length and stiffness level and the impact of rotation in the jet tokamak. Physical review letters, 102(17):175002, 2009.

[37] P. Mantica, C. Angioni, C. Challis, G. Colyer, L. Frassinetti, N. Hawkes, T. Johnson, M. Tsalas, J. Weiland, B. Baiocchi, et al. A key to improved ion core confinement in the jet tokamak: ion stiffness mitigation due to combined plasma rotation and low magnetic shear. Physical review letters, 107(13):135004, 2011.

[38] A. Di Siena, T. Görler, E. Poli, R. Bilato, H. Doerk, and A. Zocco. Resonant interaction of energetic ions with bulk-ion plasma micro-turbulence. Physics of Plasmas, 26(5):052504, 2019.

[39] P.C. Liewer. Measurements of microturbulence in tokamaks and comparisons with theories of turbulence and anomalous transport. Nuclear Fusion, 25(5):543, 1985.

[40] P.H. Diamond, S.I. Itoh, K. Itoh, and T.S. Hahm. Zonal flows in plasmas - a review. Plasma Physics and Controlled Fusion, 47:R35, 2005.

[41] F. Merz and F. Jenko. Nonlinear saturation of trapped electron modes via perpendicular particle diffusion. Physical review letters, 100(3):035005, 2008.

[42] D.R. Ernst, K.H. Burrell, W. Guttenfelder, T.L. Rhodes, A.M. Dimits, R. Bravenec, B.A. Grierson, C. Holland, J. Lohr, A. Marinoni, et al. Role of density gradient driven trapped electron mode turbulence in the h-mode inner core with electron heating. Physics of Plasmas, 23(5):056112, 2016

[43] R.E. Waltz, G.D. Kerbel, and J. Milovich. Toroidal gyro-landau fluid model turbulence simulations in a nonlinear ballooning mode representation with radial modes. Physics of Plasmas, 1(7):2229-2244, 1994.

[44] G.M. Staebler, J. Candy, N.T. Howard, and C. Holland. The role of zonal flows in the saturation of multiscale gyrokinetic turbulence. Physics of Plasmas, 23(6):062518, 2016. 